\section{La resistencia a múltiples fármacos: una amenaza para el control de la tuberculosis}

\author{
Ernesto Montoro Cardoso ${ }^{1}$
}

Palabras clave: tuberculosis resistente a múltiples drogas, terapia por observación directa.

\footnotetext{
1 Instituto de Medicina Tropical Pedro Kourí, Ciudad de La Habana, Cuba. La correspondencia debe dirigirse a: Laboratorio Nacional de Referencia e Investigaciones en Tuberculosis y Micobacterias, Instituto de Medicina Tropical Pedro Kourí, Autopista Novia del Mediodía km 612/2, La Lisa, Ciudad de La Habana, Cuba. Correo electrónico: emontoro@ipk.sld.cu.
}

El número de casos de tuberculosis en el mundo aumenta cada año en 3\% debido principalmente a la aparición y circulación de cepas resistentes a múltiples fármacos (RMF) $(1,2)$. Según los datos de la Organización Mundial de la Salud (OMS), alrededor de 50 millones de personas están infectadas por cepas de Mycobacterium tuberculosis RMF y cada año se registran 300000 nuevos casos de tuberculosis RMF. Esta situación se considera solo el comienzo de un problema de consecuencias imprevisibles, ya que la población portadora de esta infección puede ser la fuente de una epidemia de tuberculosis incontrolable (3).

La tuberculosis RMF es el resultado del tratamiento incompleto y se produce cuando los pacientes dejan de tomar los medicamentos con la regularidad prescrita o durante el tiempo requerido para eliminar la enfermedad, ya sea porque empiezan a sentirse mejor o porque los médicos y el personal de los servicios de salud prescriben los medicamentos equivocados o una combinación de fármacos inadecuada.

Desde la perspectiva de la salud pública, un tratamiento incompleto y escasamente supervisado contra la tuberculosis es peor que la ausencia de tratamiento, pues puede provocar la aparición de cepas resistentes a los medicamentos. En conclusión, se puede afirmar que en el caso de la tuberculosis, la resistencia es un reflejo fiel de las malas prácticas en el tratamiento de esta enfermedad $(4,5)$.

Hasta hace unos 50 años no había medicamentos para curar la tuberculosis. La aparición de casos de tuberculosis resistentes a fármacos se describió inmediatamente después de la introducción del tratamiento antituberculoso con estreptomicina, aunque el problema no se consideró prioritario en los países industrializados hasta la aparición de brotes de tuberculosis RMF en personas infectadas por el virus de la inmunodeficiencia humana (VIH) (6). Cuando la rifampicina y la isoniacida se convirtieron en los principales fármacos de primera línea para el tratamiento de la tuberculosis, el concepto de multirresistencia se definió como la capacidad de una cepa de $M$. tuberculosis de sobrevivir a la aplicación de estos dos medicamentos solos o combinados con algún otro fármaco. Reducir la transmisión mediante la cura de los casos de tuberculosis puede ser muy difícil si la diseminación de las cepas RMF alcanza niveles críticos (7).

La administración de la quimioterapia estandarizada de corta duración con medicamentos de 
primera línea (isoniacida, rifampicina, estreptomicina, etambutol y pirazinamida) mediante el tratamiento acortado estrictamente supervisado (TAES, más conocido por sus siglas en inglés DOTS [directly observed therapy-short course]), es la piedra angular de los actuales programas nacionales de control (PNC) de la tuberculosis. Sin embargo, los datos disponibles acerca de los resultados del tratamiento mediante estos programas indican que los pacientes con tuberculosis RMF no responden adecuadamente a la quimioterapia estandarizada de corta duración con estos medicamentos $(8,9)$.

En 1994 todavía no se conocía exactamente la magnitud del problema de la resistencia a los fármacos antituberculosos. Una revisión de la literatura científica y de informes inéditos de las últimas décadas indica que ya había altos niveles de resistencia en algunas regiones. Sin embargo, la mayoría de los estudios se realizaron con muestras que no eran representativas o sin conocerse los antecedentes terapéuticos de los pacientes participantes. Además, no había consenso acerca de las técnicas de laboratorio que se debían utilizar. Esta situación llevó a proponer la realización de investigaciones a escala internacional que determinaran la prevalencia de cepas resistentes a fármacos, aplicando conceptos, criterios y parámetros homogéneos que permitieran hacer comparaciones válidas entre los diferentes países (10).

\section{Estudios internacionales sobre la vigilancia de la resistencia a los fármacos antituberculosos}

Hasta la fecha se han realizado tres estudios internacionales dirigidos por la OMS y la Unión Internacional Contra la Tuberculosis y Enfermedades Respiratorias (UICTER). El primero comenzó en 1994 con el desarrollo de las guías técnicas de orientación que condujeron a la obtención de datos comparables entre países y regiones y a la creación de una red supranacional de laboratorios de referencia que garantizaran la calidad técnica de los estudios de susceptibilidad. Con esta última medida se buscaba avalar la confiabilidad de la información generada (11-15).

En el segundo estudio internacional se incrementó el número de países y regiones participantes y se pudo conocer mucho mejor la prevalencia de la resistencia a los fármacos antituberculosos y sus variaciones entre regiones. La elevada prevalencia de cepas RMF en algunos países refleja la estrecha relación que hay entre el control de la enfermedad y la eficacia de los PNC aplicados en el pasado.

En el tercer estudio internacional, cuyos resultados se dieron a conocer en marzo de 2004 en ocasión del Día Mundial de la Tuberculosis, abarcó a 67657 pacientes con tuberculosis provenientes de 77 países y se demostró que las cepas RMF se vuelven cada vez más resistentes y no responden a los tratamientos actuales. Se determinó, además, que $79 \%$ de los casos de tuberculosis RMF son causados por cepas resistentes a tres de los cuatro fármacos principales utilizados en el tratamiento de la tuberculosis en la actualidad (16).

Estas investigaciones demostraron la presencia de cepas de $M$. tuberculosis RMF en todas las regiones y en prácticamente todos los países estudiados, aunque aún se desconoce la verdadera magnitud del problema, especialmente en zonas que no han sido estudiadas, donde la circulación de cepas RMF puede ser aun mayor. Este estudio destaca la necesidad de ampliar la vigilancia de la resistencia a fármacos en todo el mundo (16).

Las tasas de tuberculosis resistente a diferentes fármacos son 10 veces mayores en Europa Oriental y Asia Central que en el resto del mundo, y se observa una alta concentración de casos de tuberculosis RMF en la Comunidad de Estados Independientes. Entre las 10 zonas más afectadas en todo el mundo se encuentran Estonia, Kazajstán, Letonia, Lituania, Uzbekistán y algunas regiones de la Federación Rusa, donde 14\% de los casos nuevos de tuberculosis presentaron resistencia a múltiples fármacos. En dos provincias de China se encontró que aproximadamente uno de cada 10 pacientes nuevos con tuberculosis portaban bacilos RMF. Otros países con altas tasas de tuberculosis RMF son Israel y Sudáfrica $(16,17)$.

En Europa Oriental y Asia Central, las zonas con la mayor prevalencia de tuberculosis RMF son aquellas donde las tasas de infección por el VIH han aumentado más. Las personas infectadas por el VIH son mucho más propensas a contraer las diferentes formas de tuberculosis, propagar la enfermedad rápidamente e, incluso, morir (18).

Se observó resistencia primaria ${ }^{2}$ a alguno de los fármacos individuales en 5 a $40 \%$ de los casos estudiados (mediana: 13,4\%), y resistencia primaria a múltiples fármacos en 2,2\% de los casos. La resistencia adquirida ${ }^{3}$ se detectó en $41 \%$ de los casos, mientras que la resistencia adquirida a múltiples fármacos se observó en un promedio de 13,3\% (17, 19, 20).

En las Américas, donde la situación de la tuberculosis RMF se conoce mejor, la carga de esta infección no alcanza las proporciones encontradas en otras partes del mundo (19). Según estudios inter-

\footnotetext{
2 La resistencia primaria es la observada en pacientes que no han recibido tratamiento antituberculoso anterior o que han recibido tratamiento por menos de 30 días.

3 Por resistencia adquirida se entiende la resistencia observada en pacientes que han recibido un tratamiento antituberculoso anteriormente o que han recibido tratamiento por más de 30 días.
} 
nacionales, los países de la Región con las mayores prevalencias de tuberculosis RMF son Argentina $(4,6 \%)$, República Dominicana $(6,6 \%)$ (19) y Ecuador $(6,6 \%)(17)$. No se observó un incremento significativo de las tasas de tuberculosis RMF en seis países estudiados, excepto en Perú, donde se encontró que $3 \%$ de los casos nuevos eran RMF (20).

Los países con PNC eficaces, como Chile, Cuba y Uruguay, tienen relativamente pocos casos de tuberculosis RMF. La notable reducción del número de estos casos en los Estados Unidos de América es el resultado de la introducción de medidas apropiadas para el control de la tuberculosis (especialmente gracias a la aplicación del TAES) y sobre todo el uso racional de los medicamentos de segunda línea $(15,17,21,22)$.

\section{El desarrollo de la estrategia basada en el TAES}

En la década de 1950, la elaboración de fármacos que usados en combinación y aplicados correctamente podían eliminar el bacilo de la tuberculosis y curar la enfermedad revolucionó el tratamiento y redujo drásticamente la mortalidad por tuberculosis a 5\% (23).

En la década de 1970, la introducción de la rifampicina como parte de la combinación de fármacos antituberculosos redujo la duración del tratamiento de 12 meses a 6-8 meses. Con este tratamiento acortado, los pacientes se sentían mejor más rápidamente debido al brusco descenso de la carga bacteriana durante los dos primeros meses de tratamiento intensivo. En este período, los pacientes dejaban de transmitir la enfermedad a sus familiares, amigos y compañeros de trabajo (23).

En Tanzania, en la década de 1970, el doctor Karel Styblo, de la UICTER, encabezó el desarrollo de un modelo de lucha antituberculosa basado en un enfoque gerencial de la detección y el tratamiento de los casos. En ese país, el tratamiento medicamentoso prolongado no produjo altas tasas de curación en los primeros años, por lo que se abandonó a favor del tratamiento acortado. El Programa Nacional de Lucha Antituberculosa de Tanzania fue el primero de los programas modelo de la UICTER en lograr una buena cobertura a escala nacional. Entre 1978 y 1991, la UICTER apoyó los programas de lucha antituberculosa en otros nueve países de alta prevalencia y de escasos recursos (23).

El doctor Styblo fue el primero en proponer el uso de una unidad básica de diagnóstico y tratamiento (generalmente el distrito) que contara con el personal y los recursos necesarios para diagnosticar y tratar a los pacientes, registrar su evolución e informar acerca de ella, además de gestionar los su- ministros para zonas con una población de 100000 a 150000 habitantes. El establecimiento de estas unidades básicas de tratamiento permitió integrar los diferentes elementos técnicos de la lucha antituberculosa en los servicios sanitarios generales ya existentes (23).

\section{TAES: estrategia para prevenir la resistencia}

En la actualidad la tuberculosis se puede curar. La aplicación del TAES ha pasado a ser la estrategia recomendada en todo el mundo, tanto para la cura de los pacientes como para evitar el desarrollo y la propagación de la tuberculosis RMF y para reducir la transmisión de la enfermedad. La aplicación generalizada de esta estrategia podría salvar millones de vidas $(23,24)$.

Mediante la estrategia TAES, los enfermos de tuberculosis reciben medicamentos gratuitos y permanecen bajo observación directa durante los dos primeros meses de los 6 a 8 que dura el tratamiento. De esa forma se garantiza que los pacientes tomen todos sus medicamentos, incluso en sus propios hogares $(23,24)$.

En 1993, el Banco Mundial se refirió al TAES como "una de las intervenciones de salud más eficaces en función del costo". El costo de los medicamentos para el TAES durante seis meses es de US $\$ 11,00$ por paciente en algunas partes del mundo, mientras que el costo total del retratamiento con fármacos de segunda línea es superior a US\$ 5000 por paciente y puede ascender a US\$ 7000 . Sin embargo, en la actualidad solo $30 \%$ de las personas con tuberculosis activa reciben el tratamiento según la estrategia TAES $(23,24)$.

El acceso al TAES de los enfermos con tuberculosis se ve limitado por la falta de voluntad política, la escasez de recursos, la deficiente infraestructura asistencial, la inestabilidad del suministro de medicamentos y la mala gestión, así como por el aislamiento de algunas poblaciones, la falta de viviendas y la estigmatización social de los enfermos. Estos factores impiden que muchas personas, en particular las mujeres, soliciten o tengan acceso al tratamiento $(23,24)$.

Por otra parte, estudios realizados en varios países han demostrado que hay una asociación entre la aplicación del TAES y la reducción de la resistencia a los fármacos $(15,17,25,26)$.

\section{La estrategia TAES-Plus}

La necesidad de aplicar medicamentos antituberculosos de segunda línea en algunos países con 
elevados porcentajes de RMF dio lugar al desarrollo de la estrategia TAES-Plus, basada en la aplicación de los principales componentes de la estrategia TAES $(20,27,28)$.

El objetivo principal del TAES-Plus es prevenir el desarrollo y la ulterior propagación de la tuberculosis RMF. Sin embargo, esta estrategia no es una opción universal y no es necesario impulsarla en todos los países, ya que debe aplicarse solo en las zonas donde es preciso combatir la epidemia emergente de tuberculosis RMF. El principio básico para prevenir la aparición de la resistencia a los medicamentos y para combatir la tuberculosis RMF ya establecida debe ser la implantación del TAES como primer paso. No es posible desarrollar la estrategia TAES-Plus en las zonas donde no hay un control eficaz de la tuberculosis basado en el TAES $(20,27,28)$.

La OMS y otras instituciones que participan en la lucha contra la tuberculosis han observado que la falta de acceso a los medicamentos antituberculosos de segunda línea es uno de los principales obstáculos para implantar el TAES-Plus. Por lo tanto, dentro de la nueva estrategia se ha creado un grupo de trabajo internacional dedicado a obtener precios preferenciales para los medicamentos de segunda línea que se emplearán en los proyectos piloto de TAES-Plus. Este grupo de trabajo estableció un órgano conocido como Comité de Luz Verde que revisa y aprueba los proyectos presentados por los países dirigidos a implantar la estrategia TAESPlus $(20,27,28)$.

\section{Medidas para evitar nuevos casos de tuberculosis resistente a múltiples fármacos}

La OMS y la UICTER han recomendado una serie de medidas básicas para evitar que se generen nuevos casos de farmacorresistencia múltiple $(12-16,24,29)$.

1. Recomendar tratamientos estandarizados de corta duración para todos los enfermos que tengan tuberculosis por primera vez. Siempre que exista una buena disponibilidad de medicamentos se deberán utilizar esquemas de tratamiento con isoniacida y rifampicina durante 6 meses de manera ininterrumpida. En la mayoría de los países se debe utilizar también el etambutol durante los dos primeros meses de tratamiento. La eficacia de esta medida ha sido demostrada en numerosos países y localidades donde se han logrado bajos porcentajes de resistencia a los medicamentos.

2. Recomendar el empleo de tratamientos directamente supervisados. Esta modalidad terapéutica ha demostrado que se pueden reducir las tasas de resistencia cuando el tratamiento se aplica en el marco de PNC eficaces, tanto en países con escasos recursos económicos (como Cuba y Botswana) como en países con recursos económicos medios (República Checa) o abundantes (Estados Unidos). Esta estrategia debe recomendarse para el tratamiento de todos los casos de tuberculosis en los países más pobres, y en los industrializados se debería aplicar al menos en los enfermos que pertenecen a los grupos con factores que predisponen al incumplimiento del tratamiento, como los toxicómanos, los indigentes, los alcohólicos y las personas con tuberculosis RMF o con antecedentes de haber abandonado previamente el tratamiento.

3. Administrar en una sola tableta los medicamentos antituberculosos que se pueden tomar juntos. De esta manera, si el paciente abandona el tratamiento, lo hará de forma completa y simultánea, evitándose así la aparición de cepas mutantes resistentes. La aplicación de esta medida, recomendada por la OMS y la UICTER desde 1988, ha permitido hasta la fecha disminuir eficazmente la proliferación de cepas resistentes en países como España, Brasil, Lesotho, Nueva Zelanda y Escocia. Sin embargo, esta medida no garantiza que el paciente cumpla con el tratamiento. Adicionalmente, debe establecerse un buen control de la calidad de los medicamentos asociados y de sus dosis. En particular, se recomienda la asociación de isoniacida y rifampicina, o de isoniacida, rifampicina y pirazinamida.

4. Reducir al mínimo la influencia del sector privado en el tratamiento y el control de la tuberculosis fuera de los PNC. Esta es una de las principales preocupaciones en algunos países de alta endemia, como los del Sudeste Asiático y el Pacífico Occidental -muchos de ellos tienen las mayores cargas de tuberculosis en el mundo-, donde el sector privado no sigue los lineamientos básicos de los PNC. En países como la India o Bolivia, el sector privado es en gran medida responsable de las altas tasas de resistencia observadas. Se deben fortalecer al máximo los PNC, de manera que los enfermos lleguen a confiar en las instituciones públicas y soliciten su asistencia por sí mismos. Los PNC fuertes y eficaces pueden anular la influencia descontrolada del sector privado.

5. Conseguir que el tratamiento sea totalmente gratuito para el enfermo. Esta es una de las condiciones que se deben exigir antes de establecer un PNC si se quiere garantizar su éxito. $\mathrm{Si}$ el enfermo debe pagar sus medicamentos, lo más probable es que los compre y los tome solo mientras duran los síntomas, especialmente si sus recur- 
sos económicos son reducidos, como suele ocurrir con frecuencia.

6. Implantar en cada país un PNC que abarque a toda la población y demuestre su eficacia a lo largo de los años. Los PNC deben encargarse de implantar, entre otras, las cinco medidas anteriores y con el tiempo deben lograr reducir a un mínimo la incidencia de tuberculosis RMF. Entre las experiencias con resultados satisfactorios se pueden mencionar las de Argelia, Botswana, Escocia, Lesotho, Nepal, Nueva Zelanda, República Checa y Zimbabwe, y algunas localidades de los Estados Unidos, así como las de algunos países de América Latina, como Chile, Cuba, Nicaragua, Uruguay y Venezuela.

\section{Conclusiones}

Es necesario aplicar de forma urgente medidas que eviten la aparición de más casos de tuberculosis RMF. Estas medidas solo pueden ser eficaces si se establece el control de los medicamentos de primera línea en los pacientes que no presenten resistencia a los fármacos antituberculosos, que constituyen la gran mayoría de los casos de tuberculosis. De lo contrario, es probable que aunque se disponga de fármacos de segunda línea no se logre aumentar el porcentaje de curaciones y se propague aun más la infección por bacilos RMF, con el riesgo de que la tuberculosis se convierta en una enfermedad incurable.

Según las recomendaciones de los estudios internacionales realizados, se debe dar prioridad a la vigilancia de la resistencia a los fármacos antituberculosos y a la identificación de las zonas con mayor prevalencia de tuberculosis RMF. Para evitar la aparición y la expansión de la resistencia a estos fármacos, se deben administrar combinaciones fijas de medicamentos con biodisponibilidad garantizada y los regímenes de retratamiento con fármacos de segunda línea deben utilizarse en lugares donde la tuberculosis RMF se presente en más de 3\% de los casos nuevos. Estas medidas deben aplicarse en el marco de programas bien estructurados de control de la tuberculosis basados en estrategias válidas.

\section{SYNOPSIS}

\section{Multiple drug resistance: a threat for tuberculosis control}

Drug-resistant tuberculosis (TB) was reported soon after the introduction of streptomycin, although it did not receive major attention until recently. It was not considered a major issue in the industrialized world until outbreaks of multidrug-resistant TB (MDR-TB) were reported among HIV infected people. Administration of standard shortcourse chemotherapy (SSCC) with first-line drugs under directly observed therapy (DOT) is the cornerstone of modern $T B$ control. Unfortunately, data available on the treatment outcome of MDR-TB cases under routine programmatic conditions suggest that patients with MDR-TB respond poorly to SSCC with first-line drugs.

Since 1994, the World Health Organization and the International Union Against Tuberculosis and Lung Disease (IUATLD) have conducted anti-TB drug resistance surveys through a network of subregional laboratories and researchers. Drug resistance was present in almost all settings surveyed, and prevalence varied widely across regions. High prevalence of MDR-TB is widespread in the Russian Federation and areas of the former Soviet Union (Estonia, Kazakhstan, Latvia, and Lithuania) as well as Israel, Liaoning and Henan Provinces in China, and Ecuador. The Global Project has surveyed areas representing over one third of notified TB cases. However, enormous gaps still exist in the most crucial areas. The most effective strategy to prevent the emergence of drug resistance is through implementation of the directly observed treatment short (DOTS) strategy. Effective implementation of the DOTS strategy saves lives through decreased TB transmission, decreased risk of emergence of drugresistance, and decreased risk for individual TB patients of treatment failure, TB relapse, and death. The World Bank recognizes the DOTS strategy as one of the most cost-effective health interventions, and recommends that effective TB treatment be a part of the essential clinical services package available in primary health care settings. Governments are responsible for ensuring the provision of effective TB control through the DOTS strategy.

WHO and its international partners have formed the DOTS-Plus Working Group, which is attempting to determine the best possible strategy to manage MDR-TB. One of the goals of DOTS-Plus is to increase access to expensive second-line anti-TB drugs for WHO-approved TB control programmes in low-and middle-income countries.

\section{REFERENCIAS}

1. World Health Organization. Global tuberculosis control: surveillance, planning, financing. WHO report 2004. Geneva: World Health Organization; 2004. (WHO/ HTM/TB/2004.331).

2. Hopewell PC. Tuberculosis control: how the world has changed since 1990. Bull World Health Organ. 2002;80:427.
3. Coll P. Fármacos con actividad frente a Mycobacterium tuberculosis. Enferm Infecc Microbiol Clin. 2003;21(6):299-308.

4. Bastian I, Portaels F. Multidrug-resistant tuberculosis: past, present and future. En: Bastian I, Portaels F, eds. Multidrug-resistant tuberculosis. London: Kluwer Academic Publishers; 2000. Pp. 1-12.
5. Nachega JB, Chaisson RE. Tuberculosis drug resistance: a global threat. Clin Infect Dis. 2003;36:S24-30.

6. Centers for Disease Control. Nosocomial transmission of multidrug-resistant tuberculosis among HIV-infected persons: Florida and New York, 1988-1991. MMWR. 1991;40:585-91. 
7. Súarez PG. First requirement for control of multidrug-resistant TB: realism. Bull World Health Organ. 2002;80:496.

8. Espinal MA, Jae Kim S, Súarez PG, Man Kam K, Khomenko AG, Migliori GB, et al. Standard short-course chemotherapy for drug-resistant tuberculosis. Treatment outcomes in 6 countries. JAMA. 2000;283:2537-45.

9. Pablos-Méndez A, Gowda DK, Frieden TR. Controlling multidrug-resistant tuberculosis and access to expensive drugs: a rational framework. Bull World Health Organ. 2002;80:489-94.

10. Cohn DL, Bustreo F, Raviglione MC. Drug-resistant TB: review of the worldwide situation and the WHO/IUATLD Global Surveillance Project. Clin Infect Dis. 1997;suppl 1:S121-30.

11. Bustreo F, Pablos-Méndez A, Raviglione M, Murria J, Trébucq A, Rider H. Directrices para la vigilancia de la farmacorresistencia en la tuberculosis. Ginebra: OMS/UICTER; 1997. (WHO/TB/ 96. 216).

12. World Health Organization, International Union against Tuberculosis and Lung Disease Global Project on Anti-Tuberculosis Drug Resistance Surveillance. Anti-Tuberculosis drug resistance in the world, 1994-1997. Geneva: World Health Organization; 1997. (WHO/TB/ 97. 229).

13. Pablos-Méndez A, Raviglione MC, Laszlo A, Binkin N, Rieder HL, Bustreo $\mathrm{F}$, et al. Global surveillance for antituberculosis-drug resistance, 1994-1997. N Engl J Med. 1998;338:1641-9.

14. World Health Organization, International Union against Tuberculosis and Lung Disease Global Project on AntiTuberculosis Drug Resistance Survei- llance. Anti-Tuberculosis drug resistance in the world. Report No. 2: Prevalence and trends. Geneva: World Health Organization; 2000. (WHO/CDS/TB/2000. 278).

15. Espinal MA, Laszlo A, Simonsen L, Boulahbar F, Kin SJ, World Health Organization- International Union Against Tuberculosis and Lung Disease Working Group on Anti-Tuberculous Drugs Resistance Surveillance. Global trends in resistance to anti-tuberculosis drugs. N Engl J Med. 2001;344:1294-303.

16. World Health Organization, International Union against Tuberculosis and Lung Disease Global Project on AntiTuberculosis Drug Resistance Surveillance 1999-2002. Antituberculosis drug resistance in the world. Third Global Report. Geneva: World Health Organization; 2004. (WHO/CDS/TB/2004).

17. Espinal MA. Epidemiology of multidrug-resistant tuberculosis in low- and middle-income countries. In: Bastian I, Portaels F, eds. Multidrug-resistant tuberculosis. London: Kluwer Academic Publishers; 2000. Pp. 35-48.

18. De Cock KM, Chaisson RE. Will DOTS do it? A reappraisal of tuberculosis control in countries with high rates of HIV infection. Int J Tuberc Lung Dis. 1999;3: 457-65.

19. Organización Panamericana de la Salud. Avances sobre los estudios de resistencia a fármacos antituberculosos en países de la región. Bol Tuberculosis. 2003; 6:5-6. (OPS/OMS/DPC/CD/TUB 2003).

20. Organización Panamericana de la Salud. Magnitud de la resistencia a los fármacos anti-TB en la Región de las Américas. Bol Tuberculosis. 2000;3:1-3. (OPS/ OMS/DPC/CD/TUB 2000).
21. Montoro E, Echemendía M, Lemus D, Marrero A, Llanes MJ, Valdivia JA. Surveillance of drug resistance in Mycobacterium tuberculosis: Cuba 1997-2000. Int J Tuberc Lung Dis. 2003;7(10):S199.

22. Marrero A, Caminero JA, Rodríguez R, Billo NE. Towards elimination of tuberculosis in a low income country: the experience of Cuba, 1962-1997. Thorax. 2000;55:39-45.

23. Maher D, Mikulencak M. ¿Qué es la estrategia DOTS/TAES? Ginebra: Organización Mundial de la Salud; 1999. Pp. 7-15. (WHO/CDS/CPC/TB/99.270)

24. Caminero JA. La estrategia DOTS en el control de la tuberculosis. En: Guía de la tuberculosis para médicos especialistas. París: UICTER; 2003. Pp. 239-42.

25. Styblo K, Dankova D, Drapela J. Epidemiological and clinical study of tuberculosis in the district of Kolin, Czechoslovakia: report for the first 4 years of the study (1961-64). Bull World Health Organ. 1967;37:819-74.

26. Fujiwara PI, Larkin C, Frieden TR. Directly observed therapy in New York City: history, implementation, results, and challenges. Clin Chest Med. 1997;18: $135-48$.

27. Espinal MA, Dye C, Raviglione $M$, Kochi A. Rational "DOTS plus" for the control of MDR-TB. Int J Tuberc Lung Dis. 1999;3:561-3.

28. Espinal MA, Zaleski R. TB control and access to second-line drugs: better model needed. Bull World Health Organ. 2002; 80:495-6.

29. Caminero JA. Origen, presente y futuro de las resistencias en tuberculosis. Arch Bronconeumol. 2001;37:35-42.

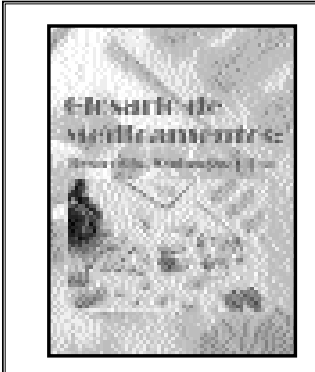

$1999 \cdot 312 \mathrm{pp}$.

ISBN 9275323054

Código: OT 118 •

Precio: US\$20.00/

US\$ 15.00 en América

Latina y el Caribe

\section{Glosario de medicamentos: desarrollo, evaluación y uso}

Este libro no es sólo una simple descripción de términos. Con el fin de facilitar al lector el uso de la literatura especializada en inglés, en él se ha incluido el equivalente, en ese idioma, de cada uno de los términos. El libro contiene, además, algunas advertencias en cuanto a las traducciones del inglés al español de términos relacionados con medicamentos, una hoja de instrucciones, una lista de abreviaturas, acrónimos, siglas, símbolos y sinónimos, así como un índice en inglés de los términos incluidos en el glosario.

Este glosario es de gran utilidad para los profesionales de la farmacología, de la medicina $\mathrm{y}$ de carreras afines que de una manera $\mathrm{u}$ otra se encuentran vinculados con organismos oficiales encargados de evaluar, registrar y seleccionar los medicamentos así como velar por su calidad e investigar su uso.

Para ordenar: http://publications.paho.org; E-mail: paho@pmds.com; Fax: (301) 209-9789; Tel: (301) 617-7806; (1-800) 472-3046 (U.S. solamente) 